\title{
The Synergistic Interaction between Probiotics and Food Affects Their Beneficial Features
}

\author{
Serena Bocchi ${ }^{1 *}$, Valeria Sagheddu ${ }^{2}$, Marina Elli ${ }^{1,2}$, Chong-Yoon Lim ${ }^{1}$, Lorenzo Morelli ${ }^{3}$ \\ ${ }^{1}$ Coree S. r. l., Piazza San Babila 5, 20122 Milan, Italy \\ ${ }^{2}$ AAT-Advanced Analytical Technologies S. r. l., Via P. Majavacca, 12, 29017 Fiorenzuola d'Arda (Piacenza), Italy \\ ${ }^{3}$ Department for Sustainable Food Processes, Faculty of Agricultural, Food and Environmental Sciences, Università \\ Cattolica del Sacro Cuore, Via Emilia Parmense, 84, 29122 Piacenza, Italy
}

Received Date: January 24, 2020; Accepted Date: January 30, 2020; Published Date: February 10, 2020

*Corresponding author: Serena Bocchi, Coree S. r. 1., Piazza San Babila 5, 20122 Milan, Italy.

Tel: +39 02 36636372; Email: serena.bocchi@coreegroup.com

\section{Abstract}

Background: Effectiveness of probiotics is conditioned by several factors. Scarce evidences currently available about the impact of the food in which or with probiotics are delivered on their beneficial features, like the adhesion to human gut. Adhesiveness plays an important role to colonize the gut, especially for specific stage of life. The aim of this work was to investigate whether the in vitro adhesiveness of lactobacilli and bifidobacteria, a mandatory prerequisite of probiotic strains, was enhanced after food matrices fermentation, focusing on weaning stage.

Methods: Probiotics were firstly evaluated for their capacity to ferment some complementary food and then for their ability to adhere to human cell lines in culture (HT-29, HT29-MTX and Caco-2) in comparison with the same strains cultured in conventional laboratory medium.

Results: Our results showed that the adhesiveness of selected probiotic strains resulted to be improved or decreased by specific weaning food, supporting the idea that probiotic strains applied to food fermentation can interact synergistically with the substrate.

Conclusions: The adhesiveness of the selected probiotic strains improved or decreased with specific weaning foods, supporting the idea that probiotic strains applied to food fermentation can interact synergistically with the substrate and therefore weaning-specific fermented functional foods could be developed.

Keywords: Adhesion; Caco-2; Fermentation; HT-29; HT29MTX; Probiotic; Weaning

\section{Introduction}

Probiotics are defined as "live microorganisms that, when administered in adequate amounts, confer a health benefit on the host" [1]. According to the FAO/WHO Joint Committee, probiotics are classified as non-pathogenic strains that should survive gastric juices, resist bile, and colonize the gastrointestinal tract [2]. The use of probiotics has been demonstrated to exert beneficial effects in terms of host wellness, in contrast to pathogenic microbes, by the production of antimicrobial compounds [3], induction of immunomodulatory effects [4], stimulation of host signaling pathways, and regulation of intestinal homeostasis [5]. The ability of probiotics to adhere to the mucosa and colonize the intestine is probably the main mechanism impacting gut health [6]. Probiotics can be administered as food supplements or through fermented food, such as traditional dairy products. Fermented foods represent a natural source of health-promoting microorganisms, usually acting as important vehicles for the delivery of lactic acid bacteria [7]. Fermentation by probiotics, instead of microbial starters, confers some benefits and therapeutic effects to humans, mainly by improving the digestibility and bioavailability of nutrients. Fermented foods are thought to be enriched in metabolites, such 
as vitamins, minerals, amino acids, and phytochemicals [8], that contribute to the health status of the consumer, stimulating the immune system, balancing the microbiota, and allowing nutrient absorption [9]. Probiotic microorganisms can increase the nutritional and functional value of food by promoting the amount and availability of nutrients and bioactive compounds originating from microbial metabolism, such as organic acids, exopolysaccharides, and conjugated linoleic acid $[\mathbf{8 , 1 0}$. Reputed scientific institutions, including the International Scientific Association for Probiotics and Prebiotics (ISAPP), are currently investigating whether and how the food matrix and its components, after fermentation, affects probiotic activity in terms of benefits for the consumer [11]. In vitro studies reported that the food matrix may affect the strain's ability to contrast pathogens [12], and one clinical trial conducted by Saxelin et al. showed that fecal populations of propionic bacteria and bifidobacteria are influenced by fermented foods metabolized by different strains [13].

FAO and WHO suggest that probiotics should survive the transit through the stomach and the gut in order to exert their beneficial effects [14]. Lipids, proteins, carbohydrates, and other components could influence probiotic viability. Therefore, food is strictly correlated to probiotic efficacy and it can be used as a deliver system to colonize the human intestine [15].

The vehicle, intended as food, is crucial for the probiotic strain survival to ensure its effects. Different food matrices can be used as probiotic delivery systems. Usually, dairy products are the most commons due to their high fat contents which protect the strain during the digestion process [16]. Actually, the market is focusing the attention on other types of food, such as fruits and vegetables. Delivery of probiotics through new functional vegetable-based food represents a very interesting opportunity for producers and consumers.

The scientific community should focus more attention on the interaction between probiotics and how their efficacy could be influenced by food matrix [17]. The ability to colonize the intestine and to adhere to the intestinal tract is fundamental properties for probiotic efficacy. This requisite is very precious in certain stages of human life, e.g. early stages, in which a proper colonisation of the gut by the microbiota represents a critical phase.

Nutrition in early life is strongly linked to health in adult life. Weaning is a transition process characterized by a gradual shift from exclusive breastfeeding or formula to the adult solid diet. The European Food Safety Authority (EFSA) guidelines recommend breastfeeding during the first 6 months of life, and weaning may begin at 4-6 months by gradually introducing complementary foods in accordance with the health status of the infant [17]. After birth, the development of the infant's microbiota is influenced by several factors, including delivery mode, breastfeeding, and geography [18]. Eubacteria colonize the intestines of newborns and have the important role of digesting complementary foods and sustaining the development of the immune system [19]. Weaning has a decisive role in the predisposition for a healthy microbiota in the baby. Thus, during the weaning diet transition, most of the bacteria normally observed during breastfeeding decrease and biodiversity increases [20]. Experimental studies have investigated important clues regarding the association between nutritional challenges in early life and disease in adulthood [21]. Tanaka et al. showed that formation of the infant gut microbiota influences the adult intestinal ecosystem and the health status of the host. Alterations in the gut microbiota during childhood may have negative effects during adult life [18]. Thus, it is clear that the infant microbiota affects the accurate development of multiple body districts. Unbalanced colonization of the gut microbiota during infancy may have a significant impact on adult metabolic disorders, such as obesity [22], allergic diseases (e.g., atopic eczema and asthma) [23], inflammatory bowel disease (IBD), and irritable bowel syndrome (IBS) [23]. Moreover, the microbiota is involved in the appropriate maturation of the endocrine and neural systems [24], with life-long consequences in neuro-psychiatric disorders, such as autism [25], depression, anxiety, stress, and sociality [26].

The WHO recommends adequate nutrition for mothers, infants, and young children with the aim of reducing infant diets that are insufficient for appropriate development of organs and systems [27]. Complementary foods with prebiotic activity have been reported to promote the release of compounds that support gut barrier maturation and stimulate the immune system [28]. Functional foods can provide metabolites that can contribute to nutritional support and minimize the risk of certain diseases [29]. Furthermore, in some countries, such as Zimbabwe, fermented weaning foods are traditionally offered to infants [30].

In light of this evidence, we aimed to investigate whether probiotic bacteria, when grown in weaning food matrices, change their in vitro gut adhesion in order to enhance their efficacy with improved colonization by beneficial microorganisms. We considered some conventional complementary foods, such as carrot, apple puree, rice cream, and oat porridge, to evaluate possible changes in terms of post-fermentation adhesiveness. Based on the hypothesis that probiotic strains applied to food fermentation can synergistically interact with the substrate, we selected newly isolated strains of Lactobacillus spp. and Bifidobacterium spp., which are able to take advantage of the interaction with their substrate and to express this synergism with improved beneficial features for the host, such as adhesiveness to the gut mucosa. Therefore, functional foods could be designed for specific requirements such as those characterizing the weaning period in order to minimize the impact of the transition from milk diet to solid foods on the human microbiota.

\section{Materials and Methods}

\section{Bacterial Strains}

Fourteen different strains of lactobacilli and bifidobacteria were used in this study. International Depositary Authority (IDA) strains were used as controls: Lactobacillus rhamnosus 
ATCC 53103, L. paracasei ATCC 334, L. plantarum DSM 9843 (299V), and Bifidobacterium animalis subsp. lactis DSM 15954 (BB12). Tested strains had different origins: B. breve LMG S-30421, L. gasseri LMG S-30423, L. gasseri LMG S30420, L. rhamnosus LMG S-30426, and L. vaginalis LMG S30427 were isolated from feces of healthy babies [30], $B$. animalis subsp. lactis LMG S-28195 from infant feces, $L$. plantarum LMG S-28194 from fruit juice, and L. plantarum
LMG S-29889, L. acidophilus LMG S-29890, and $L$. rhamnosus LMG S-29885 from wild weeds (Table 1). Lactobacilli were cultured in MRS broth (de Man Rogosa Sharpe, Difco, BD, Milan, Italy) for $24 \mathrm{~h}$ at $37^{\circ} \mathrm{C}$ and bifidobacteria in MRS broth supplemented with $0.5 \mathrm{~g} / 1$ of Lcysteine $\mathrm{HCl}$ (Sigma-Aldrich, Milan, Italy) for $48 \mathrm{~h}$ at $37^{\circ} \mathrm{C}$ under anaerobic conditions.

\begin{tabular}{|c|c|c|}
\hline Strains & Bacteria species & Origin \\
\hline LMG S-29885 & L. rhamnosus & Wild Weeds \\
\hline LMG S-30426 & L. rhamnosus & Infant Feces \\
\hline LMG S-29889 & L. plantarum & Wild Weeds \\
\hline LMG S-28194 & L. plantarum & Fruit Juice \\
\hline DSM 9843 & L. plantarum & Human Feces \\
\hline ATCC 334 & L. paracasei & Dairy Product \\
\hline LMG S-29890 & L. acidophilus & Wild Weeds \\
\hline ATCC 53103 & L. rhamnosus & Human Feces \\
\hline LMG S-30420 & L. gasseri & Infant Feces \\
\hline LMG S-30427 & L. vaginalis & Infant Feces \\
\hline LMG S-30423 & L. gasseri & Infant Feces \\
\hline DSM 15954 & B. animalis lactis & Human Feces \\
\hline LMG S-28195 & B. animalis lactis & Infant Feces \\
\hline LMG S-30421 & B. breve & Infant Feces \\
\hline
\end{tabular}

Table 1: List of bacterial strains used.

\section{Production of Weaning Food Matrices}

All food matrices were prepared separately due to their intrinsic characteristics. Raw materials were bought from local markets, kept at $4^{\circ} \mathrm{C}$, and used in the experiments within their shelf life.

\section{Carrot Juice}

After peeling, carrots were washed with tap water. Vegetables were squeezed into juice using domestic extractor. The juice was sterilized by autoclaving at $112^{\circ} \mathrm{C}$ for 30 minutes. The $\mathrm{pH}(6.8 \pm 0.2)$ was optimal for the growth of tested strains and no adjustment was needed.

\section{Apple Puree}

A commercial apple puree was chosen for the fermentation experiments. This specific food for weaning was composed of apple (99\%) and vitamin C (15 mg/100 g). Apple puree naturally has a low $\mathrm{pH}(3.5-4)$, which is not suitable for probiotic growth; therefore, the $\mathrm{pH}$ was adjusted with $\mathrm{NaOH}$ $(1 \mathrm{M})$ to $7 \pm 0.5$. The apple puree was already pasteurized when purchased, but a sterility test was performed before carrying out the experiments.

\section{Rice Cream and Oat Porridge}

Rice cream baby food and oats were purchased from the market (Italy). The label reported the following composition: rice flour (59\%) and hydrolyzed rice flour (40\%) supplemented with vitamins C and B1. Solutions of $10 \%(\mathrm{w} / \mathrm{v})$ rice flour or oats were autoclaved at $112^{\circ} \mathrm{C}$ for 30 minutes. After sterilization, the solutions were dispensed in $10 \mathrm{ml}$ tubes. To easily check the growth of bacteria suspensions, bromocresol purple (BCP, Sigma-Aldrich, Milan, Italy) was added as an acidity indicator $(0.17 \mathrm{~g} / \mathrm{L})$. The final $\mathrm{pH}$ of the rice cream and oat flakes was $6.8 \pm 0.2$ and $6.5 \pm 0.4$, respectively; therefore, no $\mathrm{pH}$ adjustment was required.

\section{Fermentation of Food Matrices}

Lactobacillus genus was first cultured in MRS broth for 18$24 \mathrm{~h}$ at $37^{\circ} \mathrm{C}$ and Bifidobacterium spp. in MRS-Cys for $48 \mathrm{~h}$ at $37^{\circ} \mathrm{C}$ under anaerobic conditions. Bacterial pellets were rinsed twice with sterile water to eliminate any residual growth medium. The absorbance of the bacterial suspensionwas adjusted to 0.7 (optical density at $600 \mathrm{~nm}, \mathrm{OD}_{600}$ ), corresponding to a theoretical value of $10^{8} \mathrm{CFU} / \mathrm{ml}$. Bacteria were inoculated in $50 \mathrm{ml}$ of food matrix media, with a final concentration in viable cells of $10^{6} \mathrm{CFU} / \mathrm{ml}$. At the end of the fermentation tests, all strains were subculture in the different 
food matrices (carrot juice, apple puree, rice cream, and oat porridge) four times before the adhesion assay was performed.

\section{Microbial Plate Counts}

Lactobacillus spp. and Bifidobacterium spp. cultured in fermented food matrices were enumerated after $24 \mathrm{~h}$ and $48 \mathrm{~h}$ in order to evaluate the growth performance. Samples were analysed by means of serial dilution and subsequent plating. One milliliter of each fermented sample was diluted 10-fold in sterile Maximum Recovery Diluent (MRD, Sigma-Aldrich, Milan, Italy) and three dilutions plated on MRS for lactobacilli or MRS-Cys for bifidobacteria, before incubating under anaerobic conditions at $37^{\circ} \mathrm{C}$ for $72 \mathrm{~h}$. After incubation, viable bacteria were counted and recorded as $\log _{10} \mathrm{CFUs}$. The $\mathrm{pH}$ values of all food matrices fermented by probiotics were measured at the end of the fermentation process using the sensION ${ }^{\mathrm{TM}} \mathrm{HACH} \mathrm{pH}$ Meter (Ghiaroni \& C, Milan, Italy).

\section{Preparation of Special Weaning Adaptation Media for Probiotic Enumeration}

Special plates were prepared to progressively adapt the probiotics to food matrices prior to the adhesion assays. Conventional growth media (MRS, MRS-Cys) were used as controls. To isolate bacterial strains on solid medium, we prepared agar plates with solid media represented by food matrices after weaning food fermentation. Carrot juice, apple puree, rice cream, and oat porridge were prepared as described above. Successively, a solution of agar $(30 \mathrm{~g} / \mathrm{L})$ was prepared and then mixed with the same volume of food, poured on Petri dishes, and then incubated for $72 \mathrm{~h}$ at $37^{\circ} \mathrm{C}$ under anaerobic conditions.

\section{Cell Lines}

Three immortalized human cell lines, considered to be representatives of various portions of the human gastrointestinal tract, were used: Caco-2, HT-29, and HT29MTX. They were obtained from the European Collection of Authenticated Cell Cultures (ECACC) and cultured in an atmosphere enriched with $5 \% \mathrm{CO}_{2}$ at $37^{\circ} \mathrm{C}$ in Dulbecco's Modified Eagle Medium (DMEM) supplemented with 10\% heat-inactivated $\left(30 \mathrm{~min}, 56^{\circ} \mathrm{C}\right)$, fetal calf serum (FCS), 50 $\mu \mathrm{g} / \mathrm{ml} \mathrm{L}$-glutamine, and $40 \mu \mathrm{g} / \mathrm{ml}$ gentamycin following the supplier's instructions (Euro Clone, Milan, Italy).

\section{Adhesion Assay}

Adhesion assays were performed by adapting the protocol previously described by Letourneau et al. [31]. Briefly, bacterial strains and cell lines were prepared in parallel. HT-29, HT29-MTX, and Caco-2 cells were rinsed with Hank's Balanced Salt Solution (HBSS, Euro Clone, Milan, Italy), trypsinized, and counted. One milliliter of the cell suspension of HT-29, HT29-MTX, and Caco-2cells was seeded at a concentration of $2.5 \times 10^{5}$ cells $/ \mathrm{ml}$ in 24 -well plates and incubated for $48 \mathrm{~h}$ (for HT-29 and HT29-MTX); Caco-2 cells were maintained for 14 days before performing the assay. For all tested conditions, three different wells were seeded with the eukaryotic cells. Three days prior to the test, all strains were streaked on both culture (MRS and MRS-Cys) and weaning food plates, prepared as indicated above, and incubated for 72 $\mathrm{h}$ at $37^{\circ} \mathrm{C}$ under anaerobic conditions. Afterwards, probiotic bacteria were directly recovered from plates, washed in sterile distilled water, and centrifuged (3000 rpm, $10 \mathrm{~min})$. The concentration of the bacterial cultures was adjusted to $10^{7} \mathrm{CFU} / \mathrm{ml}$ using a spectrophotometer (Beckman Coulter DU730 spectrophotometer, USA) at a wavelength of $600 \mathrm{~nm}$ $\left(\mathrm{OD}_{600}=0.015\right)$.

The day of the analysis, the seeded wells were washed with HBSS and incubated for $1 \mathrm{~h}$ with $875 \mu \mathrm{l}$ of DMEM $+1 \%$ FCS w/o antibiotics at $37^{\circ} \mathrm{C}$ in a $5 \% \mathrm{CO}_{2}$ atmosphere. An extra well without eukaryotic cells was inoculated with $875 \mu$ l of DMEM medium, one for each strain-condition tested as a control. The multiplicity of infection (MOI) was 5:1 (bacteria: cells). Cells were incubated with $125 \mu \mathrm{l}$ of the optically dosed bacterial suspension at $37^{\circ} \mathrm{C}$ with $5 \% \mathrm{CO}_{2}$ for $1 \mathrm{~h}$. After the incubation period, cells were washed three times with HBSS to remove unbound bacteria, inoculated with $100 \mu 1$ of trypsin-EDTA 1 X $\left(10 \mathrm{~min}\right.$ at $\left.37^{\circ} \mathrm{C}\right)$ to break the cell monolayer's, and then recovered with $900 \mu \mathrm{l}$ of MRD. Cells with adhered bacteria were diluted by 10 -fold serial dilutions in MRD and plated on MRS agar or MRS-Cys agar (lactobacilli and bifidobacteria, respectively). Bacterial cells were also incubated in wells not containing cell lines, corresponding to the $\mathrm{T} 0$ concentration in viable cells. Adhesiveness was determined as the ratio of viable probiotic cells adhering to cultured human cell lines and the number of viable bacterial cells considered at the beginning of the assay. All tested conditions were conducted in triplicate. Due to the spreading technique, the detection limit of the adhesion assay was $10^{2} \mathrm{CFU} / \mathrm{ml}$.

\section{Statistical Analysis}

Data are presented as mean \pm SD. Significance was tested by two-way ANOVA, followed by Dunnett's multiple comparisons test to analyze the effects of both the fermentation process and strains. GraphPad Prism ${ }^{\mathrm{TM}}$ software version 8.2.1 was used (GraphPad Software Inc., San Diego, California, USA). $\mathrm{P}<0.05$ was considered significant.

\section{Results}

\section{Weaning Foods are Actively Fermented by Probiotic bacteria}

Table 2 summarizes the viable counts of probiotic bacteria and $\mathrm{pH}$ values after $24 \mathrm{~h}$-incubation for lactobacilli and $48 \mathrm{~h}$ incubation for bifidobacteria. Carrot juice was fermented by all strains, with viable cell counts increasing up to $3 \log _{10}$ CFUs, with the exception of L. acidophilus LMG S-29890, L. gasseri LMG S-30420, and L. vaginalis LMG S-30427, for which the data were under the detection limit of the method. L. rhamnosus 
LMG S-30426 and L. rhamnosus ATCC 53103 had the best fermentation performance compared to the other strains, with growth values of 9.6 and $9.5 \log _{10}$ CFUs, respectively. The final concentration of B. animalis lactis DSM 15954 and B. breve
LMG S-30421 in viable cells increased up to $2 \log _{10}$ CFUs. At the end of fermentation, carrot juice fermented by probiotic microorganisms was clearly acidified by lactobacilli $(\mathrm{pH} 3.8$ 4.9) and moderately acidified by bifidobacteria ( $\mathrm{pH}$ 5.2-5.6).

\begin{tabular}{|c|c|c|c|c|c|c|c|c|c|c|c|}
\hline \multirow[t]{2}{*}{ Tested strains } & \multirow{2}{*}{$\begin{array}{c}\text { T0 } \\
\log _{10} \mathrm{CFUs}\end{array}$} & \multicolumn{2}{|c|}{$\begin{array}{r}\text { Control } \\
(\mathrm{T} 24 / 48)\end{array}$} & \multicolumn{2}{|c|}{$\begin{array}{l}\text { Carrot juice } \\
\text { (T24/48) }\end{array}$} & \multicolumn{2}{|c|}{$\begin{array}{l}\text { Apple puree } \\
\text { (T24/48) }\end{array}$} & \multicolumn{2}{|c|}{$\begin{array}{l}\text { Rice cream } \\
\text { (T24/48) }\end{array}$} & \multicolumn{2}{|c|}{$\begin{array}{c}\text { Oat porridge } \\
\text { (T24/48) }\end{array}$} \\
\hline & & $\begin{array}{l}\log _{10} \\
\text { CFUs }\end{array}$ & pH & $\begin{array}{l}\log _{10} \\
\text { CFUs }\end{array}$ & pH & $\begin{array}{l}\log _{10} \\
\text { CFUs }\end{array}$ & pH & $\begin{array}{l}\log _{10} \\
\text { CFUs }\end{array}$ & pH & $\begin{array}{l}\log _{10} \\
\text { CFUs }\end{array}$ & pH \\
\hline $\begin{array}{c}\text { L. plantarum } \\
\text { LMG S-29889 }\end{array}$ & 6.6 & 10.12 & 3.89 & 9.27 & 4.17 & 7.9 & 5.56 & 8.4 & 4.17 & 9.49 & 3.68 \\
\hline $\begin{array}{l}\text { L. plantarum } \\
\text { DSM } 9843 \\
\end{array}$ & 6.3 & 10.39 & 3.93 & 9.32 & 3.98 & 7.71 & 5.55 & 8.6 & 3.85 & 9.46 & 3.6 \\
\hline $\begin{array}{c}\text { L. plantarum } \\
\text { LMG S-28194 }\end{array}$ & 6.5 & 10.08 & 3.96 & 9.28 & 4.27 & 7.6 & 5.05 & 8.9 & 3.72 & 9.51 & 3.48 \\
\hline $\begin{array}{l}\text { L. acidophilus } \\
\text { LMG S-29890 }\end{array}$ & 6.1 & 9.86 & 4.63 & I & 4.86 & I & 5.56 & 1 & 4.25 & 8.88 & 3.68 \\
\hline $\begin{array}{c}\text { L. paracasei } \\
\text { ATCC } 334 \\
\end{array}$ & 6.2 & 10.18 & 4.12 & 9.49 & 3.98 & 7.76 & 5.67 & 8.8 & 4.04 & 9.64 & 3.44 \\
\hline $\begin{array}{l}\text { L. rhamnosus } \\
\text { ATCC } 53103\end{array}$ & 6.4 & 10.07 & 4.5 & 9.58 & 4.2 & 7.93 & 5.5 & 8.1 & 4.2 & 10.02 & 3.32 \\
\hline $\begin{array}{l}\text { L. rhamnosus } \\
\text { LMG S-30426 }\end{array}$ & 6.5 & 10.4 & 3.91 & 9.61 & 4.51 & 8.7 & 5.18 & 8.9 & 3.82 & 9.78 & 3.52 \\
\hline $\begin{array}{l}\text { L. rhamnosus } \\
\text { LMG S-29885 }\end{array}$ & 6.6 & 10.26 & 4.03 & 9.25 & 4.5 & 8.48 & 5.27 & 9.1 & 4.07 & 9.94 & 3.45 \\
\hline $\begin{array}{c}\text { L. gasseri } \\
\text { LMG S-30423 }\end{array}$ & 6.2 & 8.97 & 4.6 & 8.78 & 4.4 & 6.8 & 5.4 & 7.8 & 4.42 & 9.32 & 3.82 \\
\hline $\begin{array}{c}\text { L. gasseri } \\
\text { LMG S-30420 }\end{array}$ & 6.5 & 8.56 & 4.7 & I & 5.11 & 1 & 5.53 & 1 & 4.58 & 8.9 & 3.94 \\
\hline $\begin{array}{c}\text { L. vaginalis } \\
\text { LMG S-30427 }\end{array}$ & 5.8 & 8.96 & 4.9 & 1 & 6.35 & 6.13 & 5.93 & 8.7 & 5.47 & 8.53 & 6.03 \\
\hline $\begin{array}{c}\text { B. animalis } \\
\text { lactis DSM } \\
15954\end{array}$ & 6.1 & 8.12 & 5.2 & 7.42 & 5.98 & 6.66 & 5.57 & 7.7 & 5.12 & I & 5.45 \\
\hline $\begin{array}{c}\text { B. animalis } \\
\text { lactis LMG S- } \\
28195\end{array}$ & 6.4 & 8.23 & 5.45 & 7.24 & 6.22 & I & 6.42 & 7.2 & 5.03 & 7.7 & 5.66 \\
\hline $\begin{array}{c}\text { B. breve } \\
\text { LMG S-30421 }\end{array}$ & 6.4 & 8.22 & 5.6 & 8.21 & 5.68 & 7.14 & 6.41 & 7.8 & 5.58 & I & 5.93 \\
\hline
\end{tabular}

Table 2: Growth of probiotic bacteria ( $\log _{10} \mathrm{CFUs}$ ) and $\mathrm{pH}$ values after fermentation of weaning foods at $24 \mathrm{~h}$ for lactobacilli and $48 \mathrm{~h}$ for bifidobacteria.

Apple puree was a less suitable substrate for probiotics; the increase in viable counts was up to $2 \log _{10}$ CFUs compared to other complementary foods.

Rice cream was fermented by all examined strains, except for L. acidophilus LMG S-29890 and L. gasseri LMG S-30420. The mean growth ranged from 6 to $9 \log _{10}$ CFUs. L. rhamnosus LMG S-29885, L. rhamnosus LMG S-30426, and L. plantarum LMG S-28194 had the best performance inrice cream with 9.1, 8.9, and $8.9 \log _{10}$ CFUs, respectively, compared to $7 \log _{10}$ CFUs with L. gasseri LMG S-30423 and bifidobacteria.
The oat porridge fermentation profiles of bacterial strains, in terms of viable counts and $\mathrm{pH}$ acidification, were also investigated. Lactobacillus spp. generally metabolized this matrix, whereas bifidobacteria were not able to use this substrate, with the exception of B. animalis subsp. lactis LMG S-28195 (7.7 $\log _{10}$ CFUs). All Lactobacillus strains tested were able to acidify oat porridge, except for $L$. vaginalis LMG S30427. The $\mathrm{pH}$ values of $B$. breve $\mathrm{LMG} \mathrm{S}-30421$ and $B$. animalis lactis were weakly lower than the initial condition of $\mathrm{pH}$ 6.5. Overall results confirmed that the interaction between probiotic strains and food substrates has to be intended as 
strain-specific because distinct strains performed differently based on the type of food used as the substrate.

\section{Adhesiveness of Probiotics Grown in Food Matrices}

As reported previously, the selected strains were grown, prior to the adhesion assay, for four generations on special agar plates made with the same food on which the subsequent assays were performed. This strategy allowed us to maximize the interaction between probiotics and their substrates and to avoid false-positive results. At the same time, conventional laboratory media were used as controls.

The adhesive ability of 14 bacterial strains to HT29-MTX, HT-29, and Caco-2 cells was investigated and compared (Table 3). Our studies primarily focused on investigating the adhesiveness of lactobacilli and bifidobacteria derived from different origins and cultured in reference media. The most adhesive strain was L. rhamnosus LMG S-29885 (81.9\% to Caco-2, 79.9\% to HT-29, and77.5\% to HT29-MTX), which was isolated from plants, whereas the lowest adhesion was found in L. vaginalis LMG S-30427 (69.6\% to Caco-2, 52.5\% to HT29MTX, and value under the detection limit to HT-29). Among the strains of plant origin, L. plantarum LMG S-29889 and $L$. acidophilus LMG S-29890 demonstrated significant adhesive properties. Conversely, strains isolated from human feces exhibited slightly lower adhesive properties, especially to the mucus-producing HT29-MTX cell line. In contrast, $L$. plantarum strains, L. gasseri strains, and L. rhamnosus LMG S30426 seemed to be more adhesive to Caco-2 enterocyte-like cells, and L. rhamnosus LMG S-29885 and ATCC 53103 exhibited a similar trend of adhesion to the three cell lines. These results suggest that adhesion to human cultured cells is not strictly linked to the origin of the strain.

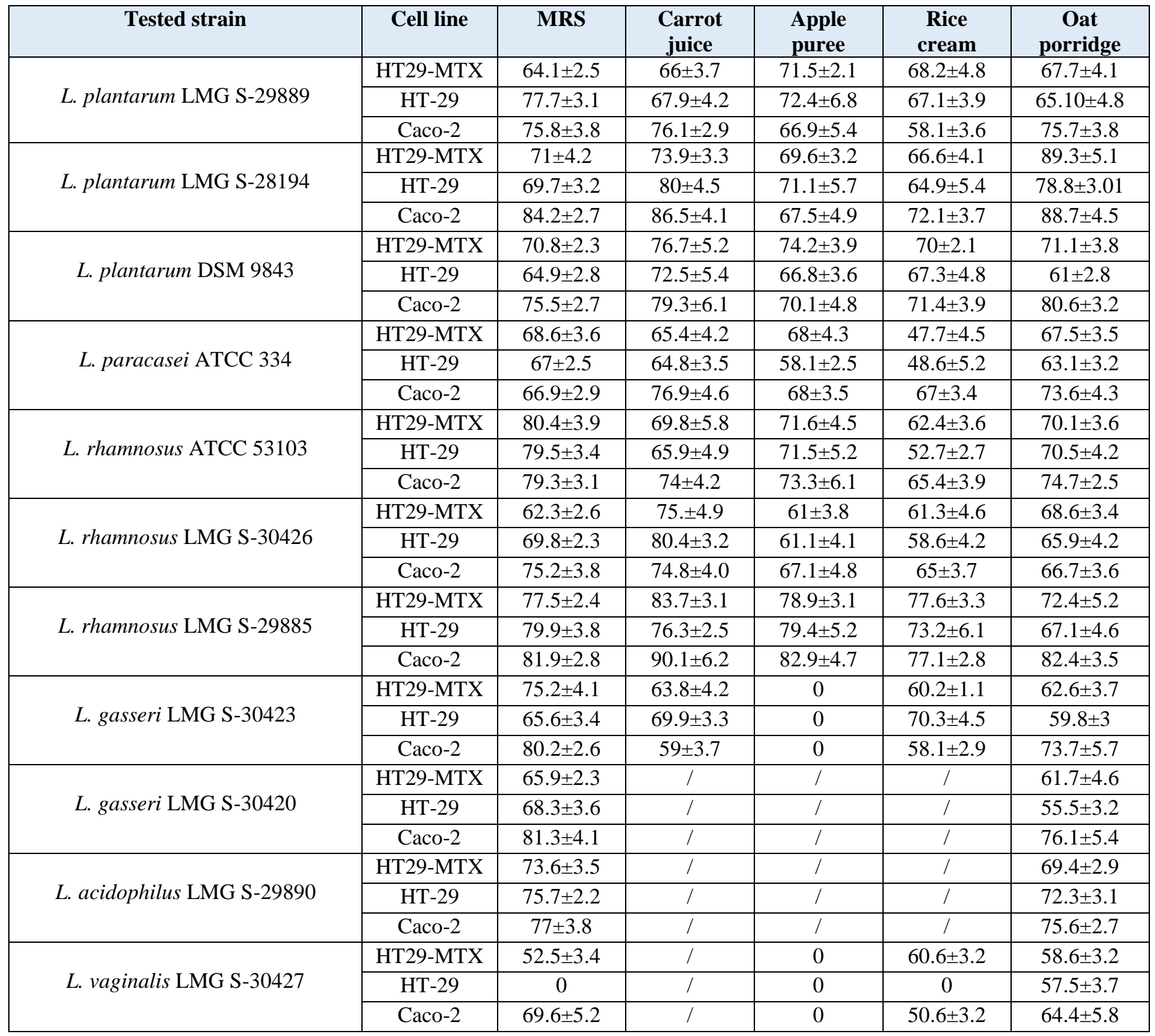




\begin{tabular}{|c|c|c|c|c|c|c|}
\hline \multirow{2}{*}{$\begin{array}{c}\text { B. animalis lactis } \\
\text { DSM 15954 }\end{array}$} & HT29-MTX & $71.6 \pm 2.6$ & $63.7 \pm 3.8$ & 0 & $63.4 \pm 4.2$ & $/$ \\
\cline { 2 - 7 } & HT-29 & $71.1 \pm 3.8$ & $63.3 \pm 5.6$ & 0 & $49.7 \pm 2.5$ & $/$ \\
\cline { 2 - 7 } & Caco-2 & $72.7 \pm 4.8$ & $72.9 \pm 4.6$ & 0 & $71.4 \pm 5.6$ & $/$ \\
\hline \multirow{3}{*}{$\begin{array}{c}\text { B. animalis lactis } \\
\text { LMG S-28195 }\end{array}$} & HT29-MTX & $73.2 \pm 3.2$ & $73.2 \pm 4.8$ & 0 & $69.6 \pm 2.8$ & $72.2 \pm 6.1$ \\
\cline { 2 - 7 } & HT-29 & $63.1 \pm 3.8$ & $69.9 \pm 3.6$ & 0 & $50.6 \pm 2.9$ & $65 \pm 5.2$ \\
\cline { 2 - 7 } & Caco-2 & $72.6 \pm 4.8$ & $81.4 \pm 4.9$ & 0 & $76.5 \pm 3.5$ & $75.1 \pm 4.2$ \\
\hline \multirow{3}{*}{$\begin{array}{l}\text { B. breve LMG S-30421 } \\
\text { HT29-MTX }\end{array}$} & $64.3 \pm 3.3$ & $78.8 \pm 2.7$ & $60.2 \pm 4.9$ & 0 & $/$ \\
\cline { 2 - 7 } & HT-29 & $75.3 \pm 4.6$ & $50.9 \pm 3.1$ & $65.3 \pm 5.1$ & 0 & $/$ \\
\cline { 2 - 6 } & Caco-2 & $74.5 \pm 2.6$ & $71.6 \pm 3.2$ & $73.3 \pm 4.6$ & 0 & $/$ \\
\hline
\end{tabular}

Table 3: Adhesion of Lactobacillus spp. and Bifidobacterium spp. to HT29-MTX, HT-29, and Caco-2 cells in reference media and after fermentation of weaning foods.

We then investigated the effect of complementary foods on the adhesion properties of each bacterial strain. The trend of results for carrot juice indicated no loss of adhesion; in general, the percentages of adhesion wavered only a few points with respect to the reference media for the three cell lines. $L$. rhamnosus LMG S-30426 and B. breve LMG S-30421 improved their adhesion to HT29-MTX $(+12.7 \%$ and $+14.6 \%$, respectively). L. gasseri LMG S-30423 recorded an evident decrease of $11.4 \%$ for HT29-MTX, decrease of $21.2 \%$ for Caco-2, and no changes for HT-29. A different strain-specific behaviour was identified with $B$. animalis subsp. lactis DSM 15954 and LMG S-28195; the first reduced its adhesiveness to HT-29 (63\%), whereas the second enhanced its adhesion $(69.9 \%)$.

The results obtained for rice cream (Figure 1C) showed a perturbation of adhesiveness; human origin L. rhamnosus ATCC 53103 and LMG S-30426 revealed a 26.8\% and $11.2 \%$ reduction in their adhesion to epithelial cells, respectively, whereas $L$. rhamnosus LMG S-29885 from plants exhibited a small reduction of $6.7 \%$. The L. plantarum strains behaved differently following rice cream fermentation. $L$. plantarum LMG S-29889 demonstrated enhanced HT29-MTX adhesion (68.2\% vs. control $64.1 \%)$ and reduced HT-29 and Caco-2 adhesion $(67.1 \%$ vs. control $77.7 \%$ and $58.1 \%$ vs. control $75.8 \%$, respectively), L. plantarum LMG S-28194 decreased its adhesion to the three cell lines, especially enterocyte-like Caco2 cells $(72.1 \%$ vs. control 84.2\%), L. plantarum DSM 9843 maintained invariant HT29-MTX adhesion but increased adhesion to HT-29 $(+2.4 \%)$ and reduced Caco-2 adhesiveness (-4.1\%). For bifidobacteria, B. breve LMG S-30421 seemed to lose its adhesiveness to the cells, but the value was under the detection limit, whereas a loss with HT-29 cells emerged for $B$. animalis lactis DSM 15954 and LMG S-28195 (49.7\% vs. $71.1 \%$ and $50.6 \%$ vs. $63.1 \%$, respectively).

The effects of incubation of Lactobacillus spp. and Bifidobacterium spp. with apple puree showed binding abilities under the detection limit for $L$. gasseri LMG S-30423, $L$. vaginalis LMG S-30427, B. animalis subsp. lactis DSM 15954, and LMG S-28195 to the three cell lines. L. plantarum strains exhibited reduced modification of adhesiveness to Caco-2 cells, whereas human origin $L$. rhamnosus spp. demonstrated a deficit of adhesion compared to L. rhamnosus LMG S-29885 isolated from plants (Figure 1B).

Furthermore, oat porridge affected adhesion properties (Table 3). L. plantarum strains LMG S-29889 and DSM 9843 did not have an altered ability to adhere to the tested cells, in contrast to L. plantarum LMG S-28194, which had increased adhesiveness (Figure 1D). L. gasseri LMG S-30423 and LMG S-30420 exhibited significant increase in adhesiveness, particularly to HT-29 (59.8\% vs. control $65.6 \%$ and $55.5 \%$ vs. $68.3 \%)$.

In general, our results indicate that food matrices affect adhesion for certain probiotic species depending on their origin. For example, the adhesion capacity of $L$. rhamnosus strains was highly affected by the origin; L. rhamnosus LMG S-29885 from plants exhibited no loss of adhesiveness independent of the tested food, whereas L. rhamnosus strains of human origin exhibited reduced adhesion following the fermentation of food. This highlights that the adhesion of probiotic strains is influenced by both the strain and the matrix (Table 3).

As HT29-MTX cells contain goblet cells that release mucins into the mucus layer, we chose to report the impact of the tested complementary foods and strains on the adhesion to HT29-MTX (Figure 1). Carrot juice (Figure 1A) and oat porridge (Figure 1B) contributed to significant enhancement of the adhesion properties of the tested strains; carrot juice significantly increased HT29-MTX adhesion of L. rhamnosus LMG S-30426 and B. breve LMG S-30421 but decreased adhesion of $L$. gasseri LMG S-30423, and oat porridge enhanced the adhesiveness of L. plantarum LMG S-28194. 
$\mathbf{A}$

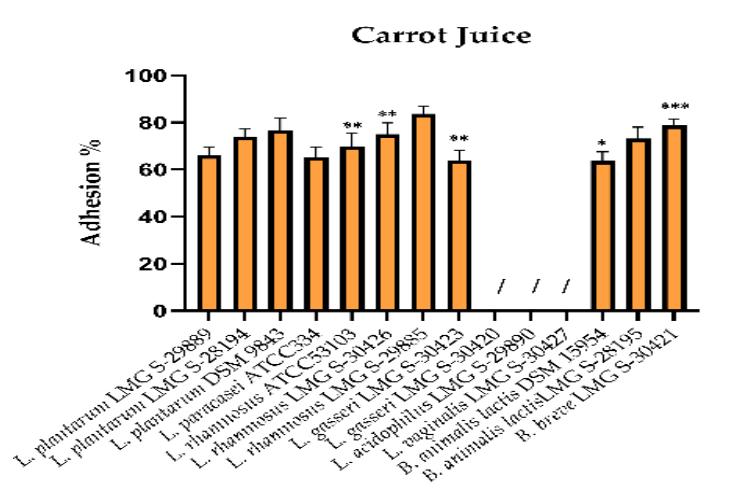

C

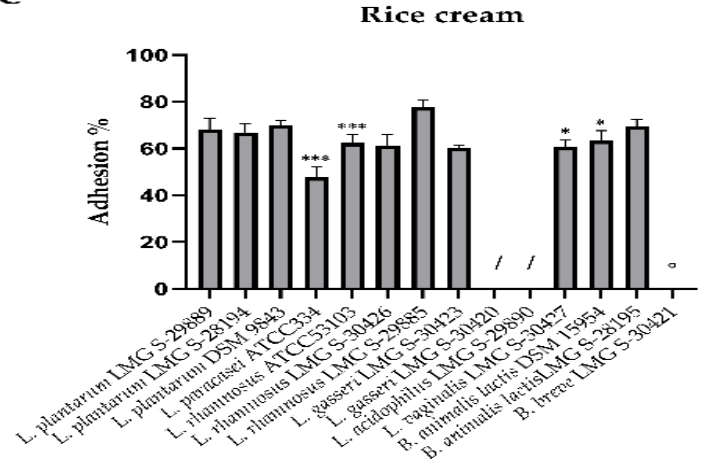

B

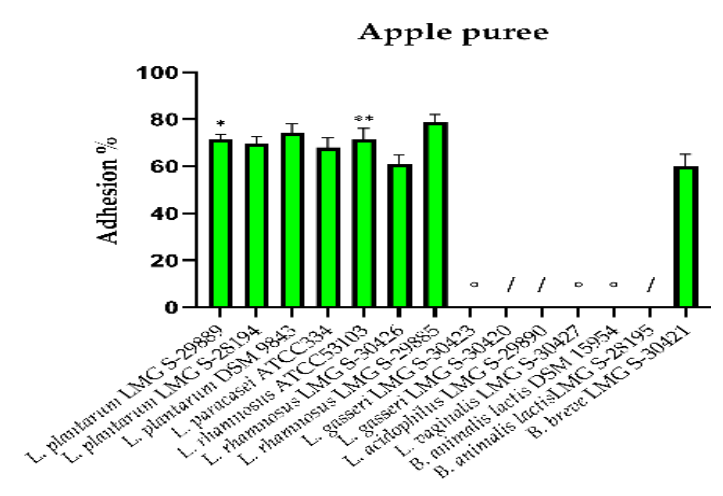

D

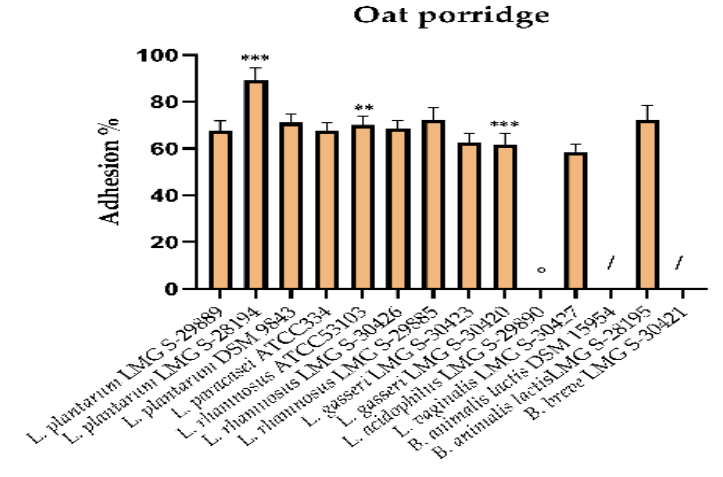

Figure 1: Impact of weaning foods on Lactobacillus spp. and Bifidobacterium spp. adhesion to HT29- MTX. (A) Cultured in carrot juice, (B) apple puree, (C) rice cream, (D) oat porridge. / indicates the inability to ferment the tested food matrix; ${ }^{\circ}$ indicates an adhesion value under the detection limit. Experiments were conducted in triplicate and expressed as mean \pm S.D. Data were analysed using a twoway ANOVA followed by Dunnett's multiple comparisons test. $* \mathrm{p}<0.05$, **p $<0.01$, ***p $<0.001$, between tested strains grown in weaning foods and the reference media (MRS and MRS-Cys).

\section{Discussion}

Weaning is an important stage of life, characterized by a progressive transition from liquid milk to solid food. Weaning foods must be rich in calories and good-quality proteins, adequate fat content, vitamins, and minerals, free from antinutritional factors and low in indigestible fibre content [33, 34]. During this crucial phase, there are important changes in both the intestinal anatomy [35] and microbiota composition [36]. Consequently, weaning foods influence the health and correct development of infants [20]. Vegetables and fruits are the first food sources used by babies to achieve the correct intake of vitamins and minerals. Moreover, probiotics are usually administered to babies as food supplements in different pharmaceutical formulations to promote and support the transition of gut microbiota during the weaning phase. However, probiotics could also be administered with weaning foods, but this opportunity has always been neglected, especially because very poor information is available about the impact of the synergistic interaction between probiotics and food.
The aim of this study was to evaluate the capacity to ferment weaning foods with probiotic strains and their ability to adhere to three lines of gastrointestinal cells when cultured in reference media. We also investigated the impact of food matrices used as fermentation substrates for probiotics on their beneficial features, mainly their adhesive properties to different types of human gut cells.

The weaning foods included in our study were selected by considering national and international paediatric and nutritional guidelines [37]. We tested weaning foods without any supplementation in order to ascertain the real capacity of the tested matrix to support single strains. In agreement with previous studies, carrot juice, apple puree, rice cream, and oat porridge were fermented by lactobacilli and bifidobacteria [3840]. Our results show good growth performances for most of the tested strains, except for L. gasseri LMG S-30420 and $L$. acidophilus LMG S-29890 that were able to ferment and survive only in oat porridge. The capacity to ferment food matrices seemed to be independent of the origin of the strains; L. plantarum LMG S-29889, LMG S-28194, and DSM 9843, 
which were isolated from different sources, exhibited the same ability to grow in the same substrate. Similar trends were recorded for L. rhamnosus LMG S-29885, LMG S-30426, and ATCC 53103 that showed comparable behavior even if isolated from different environments. The ability to ferment weaning foods did not correlate with the species; L. gasseri LMG S30423 and L. gasseri LMG S-30420 isolated from the same source exhibited different performances in tested food matrices, except oats. This phenomenon suggested that the capacity to ferment food matrices was strain-specific, supporting the idea that each strain, even those of the same species, presented certain characteristics that made them different from other strains [1]. In particular, the capacity to ferment food matrices can be a property of bacterial strains, but Marteau also showed that microbial strains from the same species can be dissimilar in their genotype, phenotype, and properties [41]. Moreover, ISAPP recently considered that some beneficial properties can be clustered as genus-, species-, and strain-specific. Aspects related to clinical efficacy are recognized as being peculiar of single strains, whereas other aspects are considered widespread [1].

Adhesion of probiotic strains to the mucosal surface has been considered one of the initial event sin successful colonization of the host gastrointestinal tract. Adhesion mechanisms can be due to weak (van der Waals forces, hydrophobicity, and ionic bounds) [42] and covalent interactions (cell wall-anchored proteins such as adhesins, pili (fimbriae), and others) [43]. Adhesion abilities are intrinsic to the single bacterial strain [44], and biological markers such as cell surface proteins, exopolysaccharides, and lipoteichoic acid have been associated with this phenomenon [4244]. Evidence indicates that $L$. gasseri, L. plantarum, $L$. paracasei, L. rhamnosus, L. acidophilus, L. vaginalis, B. breve, and $B$. animalis lactis are able to adhere to the gastrointestinal cells via different mechanisms [45-48]. Probiotics can exert their positive effects both transiently, not colonizing the intestine or long-term by adhering to the intestine. The three cell lines (HT29, HT29-MTX, and Caco-2) in this study are derived from the epithelial colorectal adenocarcinoma, the most representative for in vitro models of adhesion [49]. Although some studies have been published regarding fermented food and the adhesive properties of probiotic strains, there has been a lack of investigation about how food matrices could influence the adhesiveness of microorganisms. Strains grown in the reference media had good performance adhering to the three cell lines. For the normal growth media, the percentage of adhesion of strains to enterocyte-like Caco-2 cells was higher than adhesion to the mucus-secreting HT29-MTX intestinal cells. The only value under the detection limit was recorded for L. vaginalis LMG S30427 to HT-29. Little has been published about the adhesive capacity of L. vaginalis strains on HT-29, even if Martin et al. recorded a good percentage of adhesion [46]. Unfortunately, it is difficult to compare our data with other research due to the diverse adhesion assay approaches influenced by many factors, including medium growth, $\mathrm{pH}$, and bacterial concentration [50]. Nevertheless, our data suggest that adhesion properties are straindependent and not species-dependent because probiotic bacteria have different strategies to adhere to the gastrointestinal cells [51]. L. rhamnosus LMG S-30426 and L. rhamnosus LMG S-
29885 had incomparable percentages of adhesion to the three cell lines we used, even though they belonged to the same species. In addition, L. plantarum spp. exhibited different behaviours when incubated with the gastrointestinal cells. The data indicated patterns of adhesion different for each strain, probably due to the different expression of ligands on the cell surfaces of HT-29, HT29-MTX, and Caco-2cells [52]. The ecological origin of the strains seemed not to influence the adhesive properties when cultured in reference media. L. rhamnosus LMG S-30426 and $L$. rhamnosus LMG S-29885, derived from humans and plants, respectively, demonstrated relatively good adhesive properties with behaviour particular to each cell line, regardless of the origin. This event suggests that the origin may affect their capacity for adhesion [53], but the provenience of the strain is not a limit for adhesiveness to cells. L. rhamnosus LMG S-29885 had higher percentages of adhesion than L. rhamnosus LMG S30426. These data support the hypothesis that mechanisms of probiotic adhesion are influenced mainly by strain-specificity, but also by the selected in vitro cell models. Moreover, different strain origins seemed not to influence the adhesiveness to human cells.

The second step was to evaluate the effects of weaning foods on strain adhesiveness. With regards to apple puree, our data show that the adhesion of several Lactobacillus spp. was affected, especially those of human origin (L. gasseri LMG S30423 and LMG S-30420, L. vaginalis LMG S-30427, and bifidobacteria). For L. rhamnosus spp., we observed a decrease in adhesiveness; the human origin strains L. rhamnosus LMG S-30426 and ATCC 53103 reduced adhesion, whereas the plant origin L. rhamnosus LMG S-29885 seemed to not be affected. In addition, all tested L. plantarum strains slightly increased their adhesiveness despite their source. In agreement with our data, Sredkova et al. found that lactobacilli pre-treated with pectin, a typical compound in apples influenced their mechanisms of adhesion, exhibiting a loss of adhesive properties [54].

Tamminen et al. reported that in vitro adhesion of $L$. rhamnosus ATCC 53103 to human colon mucus decreased after fermentation in carrot juice [55]. Our results suggest that increased adhesion can be attributable not to physical bonds, but to other interactions, such as those between the strain and intestinal cells. In addition, rice cream reduced the adhesion capacity of most of the tested strains, whereas the oat-based medium had positive effects on $L$. plantarum adhesion to the three cell lines.

Several reviews have indicated that the composition of the growth medium changes the adhesion of probiotics $[\mathbf{5 6 , 5 7 ]}$. For example, Ranadheera et al. observed that the adhesion of each probiotic strain to Caco-2 cells is dependent on the type of yogurt used in the assay compared to the normal condition used in their experiments [58]. A bacterial strain can produce exopolysaccharide with different rheological properties, exploiting the different carbon sources present in the medium [59]. Furthermore, stress conditions can alter cell surface properties. For example, L. rhamnosus ATCC 53103 down regulates the exopolysaccharide gene in response to bile [59]. 
Both the manufacturing process and food matrix influence probiotic adhesion properties and the exclusion of pathogens [12]. These changes could be due to alterations in the bacterial surface, including hydrophobic characteristics, or depend on various factors, such as culture media, growth phase, temperature, and $\mathrm{pH}[\mathbf{6 0}]$. In line with our results, Aissi et al. reported that the adhesion of bifidobacteria to intestinal mucosa could be influenced by environmental factors [48]. However, few studies have focused only on the effects of food matrices in sustaining strain growth during fermentation and protecting it during digestion in vitro [61], regardless of the adhesion properties. Thus, a specific strain could be associated with a food matrix, not only for its delivery and survival during gastrointestinal transit, but also to foster intestinal colonization. In light of the evidence, food matrix could modulate different events exerted by the strain on the host, finally resulting in mechanisms of synergy. Our findings also suggest that the effect of food matrix on the adhesiveness is strain-specific, and some vegetable food could positively or negatively affect the adhesive properties. In particular, the fermentation of weaning foods resulted in a synergistic effect on the adhesion of $L$. rhamnosus LMG S-30426 and B. breve LMG S-30421. During fermentation, bacterial strains produce a wide range of metabolites, some of which are bio available and bioactive with healthy properties, that can be absorbed by the gastrointestinal cells and exert their effect at the systemic level [62]. A metabolomic study conducted by Tomita et al. provided information on the different functional and nutritional properties of fermented food in the presence of several Lactobacillus spp. in terms of metabolites [63]. In order to investigate these unique interactions between food and probiotic strains in more detail, we decided to move towards a metabolomic approach to identify peculiar bioactive metabolites generated by the probiotic fermentation of weaning foods.

\section{Conclusion}

The mechanism of the interactions between complementary food and probiotics can be speculated. Beneficial bacteria could be a valid support for the transient phase of gut colonization during the weaning stage in order to improve the synergistic effect on the host. Accurate selection of specific strains combined with food matrices could promote acid and bile tolerance during digestion, and affect their adhesiveness to the gut mucosa.

\section{Author Contributions}

\begin{tabular}{|c|c|}
\hline Author & Contribution \\
\hline Serena Bocchi & Investigation, Validation, Formal analysis, Data curation, Writing. \\
\hline Valeria Sagheddu & Validation, Methodology, Data curation, Writing; supervision. \\
\hline Marina Elli & $\begin{array}{c}\text { Conceptualization, Methodology, Editing, Supervision, Validation, Data } \\
\text { curation, Writing. }\end{array}$ \\
\hline Lorenzo Morelli & Supervision, Validation, Writing; Editing. \\
\hline Chong-Yoon Lim & Resources, Project administration. \\
\hline \multicolumn{2}{|c|}{ Funding: This research received no external funding. } \\
\hline
\end{tabular}

Conflicts of Interest: The authors declare no conflict of interest.

\section{References}

1. Hill C, Guarner F, Reid G, Gibson GR, Merenstein DJ, et al. (2014) Expert consensus document: The international scientific association for probiotics and prebiotics consensus statement on the scope and appropriate use of the term probiotic. Nature. Reviews: Gastroenterology and Hepatology 11: 506-514.

2. Food and Agriculture Organization/ World Health Organization. Guidelines for the Evaluation of Probiotics in Food London (April 2002).

3. Saxelin M, Tynkkynen S, Mattila-Sandholm T, De Vos WM (2005) Probiotic and other functional microbes: From markets to mechanisms. Current Opinion Biotechnology 16: 204-211.

4. Boirivant M, Strober W (2007) The mechanism of action of probiotics Current Opinion Gastroenterology 23: 679692.

5. Van Baarlen P, Wells JM, Kleerebezem M. (2013) Regulation of intestinal homeostasis and immunity with probiotic lactobacilli. Trends in Immunology 34: 208-215.

6. Monteagudo-Mera A, Rastall RA, Gibson GR, Charalampopoulos D, ChatzifragkouA (2019) Adhesion mechanisms mediated by probiotics and prebiotics and their potential impact on human health. Applied Microbiology and Biotechnology 103: 6463-6472.

7. Rivera-Espinoza Y, Gallardo-Navarro Y (2010) Non-dairy probiotic products. Food Microbiology 27: 1-11.

8. Di Cagno R, Filannino P, Gobbetti M (2016) Novel Fermented Fruit and Vegetable-Based Products. Food Engineering Series. Springer: Cham, pp 279-291.

9. Zhao W, Liu Y, Latta M, Ma W, Wu Z, et al. (2019) Probiotics database: a potential source of fermented foods. International Journal of Food Properties 22: 197-216.

10. Leroy F, De Vuyst L (2014) Fermented food in the context of a healthy diet: How to produce novel functional foods? Current Opinion in Clinical Nutrition and MetabolicCare17: 574-581.

11. International Scientific Association for Probiotics and Prebiotics. Effects of the food matrix on probiotics' 
efficacy: how much should we care? 2019.

12. Grześkowiak $L$, Isolauri $E$, Salminen $S$, Gueimonde $M$ (2011) Manufacturing process influences properties of probiotic bacteria. British Journal of Nutrition105: 887894.

13. Saxelin M, Lassig A, Karjalainen H, Tynkkynen S, Surakka A, et al. (2010) Persistence of probiotic strains in the gastrointestinal tract when administered as capsules yoghurt or cheese. International. Journal of Food Microbiology 144: 293-300.

14. Terpou A, Papadaki A, Lappa IK, Kachrimanidou V, Bosnea LA, et al. (2019) Probioticsin Food Systems: Significance and Emerging Strategies towards Improved Viability and Delivery of Enhanced Beneficial Value. Nutrients 11.

15. Ranadheera RDCS, Baines SK, Adams MC (2010) Importance of food in probiotic efficacy. Food Research International 43:1-7.

16. Meybod NM, Mortazavian AM (2017) Probiotic Supplements and Food Products: A Comparative Approach Biochemistry and Pharmacology 6: 2.

17. Castenmiller J, de Henauw S, Hirsch-Ernst K, Kearney J, Maciuk A, et al. (2019) Appropriate age range for introduction of complementary feeding into an infant's diet. EFSA Journal 17: 5780.

18. Tanaka M, Nakayama J (2017) Development of the gut microbiota in infancy and its impact on health in later life. Allergology International 66: 515-522.

19. Stark PL, Lee A (1982) The microbial ecology of the large bowel of breast-fed and formula-fed infants during the first year of life. Journal of Medical Microbiology15: 189-203.

20. Fallani M, Amarri S, Uusijarvi A, Adam R, Khanna S, et al. (2011) Determinants of the human infant intestinal microbiota after the introduction of first complementary foods in infant samples from five European centres. Microbiology 157: 1385-1392.

21. Langley-Evans SC (2015) Nutrition in early life and the programming of adult disease: A review. Journal of Human Nutrition and Diet 1: 1-14.

22. Mohammadkhah AI, Simpson EB, Patterson SG, Ferguson JF (2018) Development of the Gut Microbiome in Children and Lifetime Implications for Obesity and Cardiometabolic Disease. Children 5: 160.

23. Stiemsma LT, Michels KB (2018) The Role of the microbiome in the developmental origins of health and disease. Pediatrics 141.

24. Zuo T, Ng SC (2018) The Gut Microbiota in the Pathogenesis and Therapeutics of Inflammatory bowel disease. Frontier in Microbiology 9: 2247.

25. Yang I, Corwin EJ, Brennan PA, Jordan S, Murphy JR, et al. (2016) The infant microbiome: Implications for infant health and neurocognitive development. Nursing Research 65: 76-88.

26. Sordillo JE, Korrick S, Laranjo N, Carey V, Weinstock GM, et al. (2019) Association of the Infant Gut Microbiome with Early Childhood Neurodevelopmental Outcomes. JAMA Network Open 2: e190905.

27. World Health Organization. Global nutrition targets 2025: low birth weight policybrief(WHO/NMH/NHD/14.5).
Geneva: WHO (2014)

28. McKeen S, Young W, Mullaney J, Fraser K, McNabb WC, et al. (2019) Infant complementary feeding of prebiotics for the microbiome and immunity. Nutrients 11: 364 .

29. Getahun A, Tesfaye A, Muleta D (2016) Investigation of the Potential Benefits and Risks of Probiotics and Prebiotics and their Synergy in Fermented Foods. Singapore Journal of Chemical Biololy 6: 1-16.

30. Simango C (1997) Potential use of traditional fermented foods for weaning in Zimbabwe. Social Science \& Medicine 44: 1065-1068.

31. Zhang D, Zhang S, Guidesi E, Zonenschain D, Sagheddu V, et al. (2018) Isolation and Characterization of New Probiotic Strains from Chinese Babies. Journal of Clinical Gastroenterology 52 Suppl1.

32. Letourneau J, Levesque C, Berthiaume F, Jacques $M$, Mourez M (2011) In Vitro Assay of Bacterial Adhesion onto Mammalian Epithelial Cells. Journal of Visualized Experiments16: pii: 2783.

33. Pan American Health Organization. Guiding Principles for Complementary Feeding of the Breastfed (2001).

34. World Health Organization. Guiding Principles for Feeding Non-Breastfed Children6-24Months of Age (2005).

35. Xun W, Shi L, Zhou H, Hou G, Cao T (2018) Effect of weaning age on intestinal mucosal morphology permeability gene expression of tight junction proteins cytokines and secretory $\operatorname{IgA}$ in Wuzhishan mini piglets. Italian Journal of Animal Science 17.

36. Andreas NJ, Kampmann B, Mehring Le-Doare K (2015) Human breast milk: A review nits composition and bioactivity. Early Human Development 91: 629-635.

37. Alvisi P, Brusa S, Alboresi S, Amarri S, Bottau P, et al. (2015) Recommendations on complementary feeding for healthy full-term infants. Italian Journal of Pediatrics 41: 36.

38. Li C, Nie SP, Zhu KX, Xiong T, Xie MY (2016) Lactobacillus plantarumNCU116fermented carrot juice evokes changes of metabolites in serum from type 2 diabetic rats. Food Research International 80: 36-40.

39. Hu R, Zeng F, Wu L, Wan X, Chen Y, et al. (2019) Fermented carrot juice attenuates type2diabetes by mediating gut microbiota in rats. Food \& Function 10: 2935-2946.

40. Ogué-Bon E, Khoo C, Hoyles L, McCartney AL, Gibson $\mathrm{GR}$, et al. (2011) In vitro fermentation of rice bran combined with Lactobacillus acidophilus 14 150B or Bifidobacterium longum 05 by the canine faecal microbiota. FEMS Microbiology Ecology 75: 365-376.

41. Marteau P (2011) Evidence of Probiotic Strain Specificity Makes Extrapolation of Results Impossible From a Strain to Another Even From the Same Species. Annals of Gastroenterology \& Hepatology.

42. Celebioglu H, Svensson B (2018) Dietary Nutrients Proteomes and Adhesion of Probiotic Lactobacilli to Mucin and Host Epithelial Cells. Microorganisms 21: 6.

43. Nishiyama K, Sugiyama M, Mukai T (2016) Adhesion Properties of Lactic Acid Bacteria on Intestinal Mucin. Microorganisms 4: 34. 
44. Lebeer S, Vanderleyden J, De Keersmaecker SCJ (2008) Genes and Molecules of Lactobacilli Supporting Probiotic Action. Microbiology and Molecular Biology Reviews 72: 728-764.

45. Arai T, Obuchi S, Eguchi K, Seto Y. (2016) In vitro investigation of molecules involved in Lactobacillus gasseri SBT2055 adhesion to host intestinal tract components. Journal of Applied Microbiology 120: 16581667.

46. Martín R, Sánchez B, Suárez JE, Urdaci MC. (2012) Characterization of the adherence properties of human Lactobacilli strains to be used as vaginal probiotics. FEMS Microbiology Letters 328: 166-173.

47. Lebeer S, Claes I, Tytgat HLP, Verhoeven TLA, Marien E, et al. (2012) Functional analysis of Lactobacillus rhamnosus GG pili in relation to adhesion and immunomodulatory interactions with intestinal epithelial cells. Applied and Environmental Microbiology 78: 185193.

48. Aissi EA, Lecocq M, Brassart C, Bouquelet S (2001) Adhesion of some Bifidobacterial strains to human enterocyte-like cells and binding to mucosal glycoproteins. Microbial Ecology in Health and Disease 13:1.

49. Gagnon M, ZihlerBerner A, Chervet N, Chassard C, Lacroix C (2013) Comparison oftheCaco-2 HT-29 and the mucus-secreting HT29-MTX intestinal cell models to investigate Salmonella adhesion and invasion. Journal of Microbiological Methods 94: 274-279.

50. Laine R, Salminen S, Benno Y, Ouwehand AC (2003) Performance of bifidobacteria in oat-based media. International Journal of Food Microbiology 83: 105-109.

51. Wang G, Xia Y, Cui J, Gu Z, Song Y, et al. (2014) The Roles of Moonlighting Proteins in Bacteria. Current issues in molecular biology 16: 15-22.

52. Furrie E, Macfarlane S, Thomson G, Macfarlane GT. (2005) Toll-like receptors-2 -3 and-4expression patterns on human colon and their regulation by mucosal-associated bacteria. Immunology 115: 565-574.

53. Vemuri R, Shinde $T$, Shastri MD, Perera AP, Tristram $S$, et al. (2018) A human origin strain Lactobacillus acidophilus DDS-1 exhibits superior in vitro probiotic efficacy in comparison toplantor dairy origin probiotics. International Journal of Medical Science 15: 840-848.

54. Sredkova P, Iliev I, Dzhambazov B, Batsalova T (2017) Prebiotic Treatment Influence the Adhesion Properties of
Three Lactobacillus strains. International Journal of Current Microbiology and Applied Sciences 6: 2914-2924.

55. Tamminen M, Salminen S, Ouwehand AC (2013) Fermentation of Carrot Preservation of Adhesion Juice by Probiotics: Viability and Preservation of Adhesion. International Journal of Biotechnology for Wellness Industries 2: 10-15.

56. Ouwehand AC, Salminen S (2003) In vitro Adhesion Assays for Probiotics and their in vivo Relevance: A Review. Microbial Ecology in Health and Disease 15: 175184.

57. Deepika G, Karunakaran E, Hurley CR, Biggs CA, Charalampopoulos D (2012) Influence of fermentation conditions on the surface properties and adhesion of Lactobacillus rhamnosus GG. Microbial Cell Factories 11:116.

58. Ranadheera CS, Evans CA, Adams MC, Baines SK (2012) In vitro analysis of gastrointestinal tolerance and intestinal cell adhesion of probiotics in goat's milk ice cream and yogurt. Food Research International 49: 619-625.

59. Polak-Berecka M, Choma A, Wäsko A, Górska S, Gamian A, et al. (2015) Physicochemical characterization of exopolysaccharides produced by Lactobacillus rhamnosus on various carbon sources. Carbohydrate Polymers 117: 501-509.

60. Koskenniemi K, Laakso $\mathrm{K}$, Koponen J, Kankainen M, Greco D, et al. (2011) Proteomics and transcriptomics characterization of bile stress response in probiotic Lactobacillus rhamnosus GG. Molecular \& Cellular Proteomics 10: M110.002741.

61. Kankaanpää PE, Salminen SJ, Isolauri E, Lee YK. (2001) The influence of polyunsaturated fatty acids on probiotic growth and adhesion. FEMS Microbiology Letters 194: 149-53.

62. Peters A, Krumbholz P, Jäger E, Heintz-Buschart A, Çakir MV, et al. (2019). Metabolites of lactic acid bacteria present in fermented foods are highly potent agonists of human hydroxycarboxylic acid receptor 3. PLOS Genetics 15: e1008283.

63. Tomita S, Saito K, Nakamura T, Sekiyama Y, Kikuchi J (2017) Rapid discrimination of strain-dependent fermentation characteristics among Lactobacillus strains by NMR-based metabolomics of fermented vegetable juice. PLoS One 12: e0182229.

Citation: Bocchi S, Sagheddu V, Elli M, Lim CY, Morelli L (2020) The Synergistic Interaction between Probiotics and Food Affects Their Beneficial Features. Adv Nutri and Food Scie: ANAFS-165. 